\title{
Increase of Fatty Acid Oxidation and VLDL Assembly and Secretion Overexpression of PTEN in Cultured Hepatocytes of Newborn Calf
}

\author{
Shixin Fu ${ }^{a, b}$ Qinghua Deng ${ }^{a}$ Wengtao Yang ${ }^{a}$ Hongyan Ding ${ }^{a}$ Xiaoxu Wang ${ }^{a}$ \\ Peng $\mathrm{Li}^{\mathrm{c}}$ Xinwei $\mathrm{Li}^{\mathrm{a}}$ Zhe Wang ${ }^{\mathrm{a}}$ Xiaobing $\mathrm{Li}^{\mathrm{a}}$ Guowen Liu \\ aCollege of Animal Science and Veterinary Medicine, Jilin University, 5333 Xi'an Road, Changchun; \\ 'DInstitute of Animal Science and Technology, Heilongjiang Bayi Agriculture University, Daqing, \\ Heilongjiang; 'College of Animal Science and Veterinary Medicine, Shenyang Agricultural University, \\ Shenyang, Liaoning
}

\section{Key Words}

PTEN • Fatty liver • Fatty acid oxidation • VLDL

\begin{abstract}
Background: Phosphatase and tensin homolog (PTEN) is a potent tumor suppressor gene that also plays a vital role in regulating fatty acid metabolism. Here we attempted to elucidate the role of PTEN in the regulation of fatty acid oxidation and the assembly and secretion of very low density lipoprotein (VLDL) in dairy cow liver. Methods: We transfected primary culture calf hepatocytes with adenovirus-mediated PTEN overexpression vector (AD-GFP-PTEN).PTENoverexpressing hepatocytes and control hepatocytes were obtained. Results: Compared with controls, overexpression of PTEN significantly up-regulated CPT I, ACSL, HADH expression $(p<0.05)$, which are all involved in fatty acid oxidation. At the same time, the expression of ApoB100 $(p<0.01)$, ApoE $(p<0.05)$ and MTP $(p<0.01)$ increased. Therefore, the assembly and secretion of VLDL was enhanced $(p<0.05)$. The expression of LDLR was slightly up-regulated, but there was no significant difference $(p>0.05)$. To demonstrate that fatty acid metabolism was changed, we measured the concentrations of TG and VLDL. The concentration of TG was significantly decreased in hepatocytes $(p<0.01)$, while the concentration of VLDL was significantly increased in the medium $(P<0.05)$. Conclusions: Overexpressing PTEN enhanced fatty acid oxidation and assembly and secretion of VLDL. PTEN gene therapy could have therapeutic potential for fatty liver diseases of dairy cattle.
\end{abstract}




\section{Introduction}

Fatty liver of dairy cattle is a common metabolic disorder in the perinatal period. It has become the most significant dairy cattle disease in the world $[1,2]$. The most important factor in the induction of hepatic lipidosis is negative energy balance (NEB) [3]. The clinical feature of fatty liver is the excessive deposition of fat in the liver. In recent research, when dairy cattle were fed high-energy food in the perinatal period, the blood plasma concentration of nonesterified fatty acids (NEFA) rapidly became higher after parturition, and the liver TG content increased. But also ruminants have little ability to secrete VLDL from the liver, making them susceptible to fatty liver disease $[4,5]$. Accumulation of TG in the liver occurs, and fatty liver is consequently induced [6-8]. Ketone bodies increased in blood plasma, causing ketosis. Capability of fatty acid oxidation and assembly, and the secretion of VLDL, are the most important factors causing fatty liver disease in dairy cattle. In our previous study, we showed that the expression of CPT I, ApoB100, HADH mRNA, oxaloacetic acid (OAA) and the density of VLDL decreased in the liver tissue and serum of periparturient dairy cattle $[9,10]$, as reported by U. Bernabucci [11]. We observed that the capability of fatty acid oxidation was degraded and the assembly and secretion of VLDL were deficient in cattle with fatty liver disease; therefore, they are important factors contributing to fatty liver disease in dairy cattle.

PTEN, a tumor suppressor gene $[12,13]$, has been shown to play a vital role in different signal transduction pathways by inhibiting the proliferation and migration of tumor cells [14], inducing apoptosis of tumor cells and retaining the normal physiological functions of cells $[15,16]$. Most studies on PTEN mechanisms have focused on tumor suppression. However, recently many researchers have discovered that PTEN also plays important roles in the development of fatty liver disease via lipid phosphatase, protein phosphatase and phosphatase-independent activities.

In a recent study, lipid metabolism was shown to be regulated by insulin in the liver, which is accomplished through cell surface receptors and regulatory factors such as phosphatidylinositoI3-kinase (PI3-kinase) and serine-threonine kinase. The insulin signal transduction pathway is activated by protein hydroxyphenylaminopropionic acid kinase or lipid phosphatidase. Insulin signaling in the liver leads to the accumulation of phosphatidylinositol-triphosphate $\left(\mathrm{PIP}_{3}\right)[17,18]$. Deletion of the PTEN gene can increase $\mathrm{PIP}_{3}$ levels and lead to fatty liver development [19]. However, it is unknown whether the capability of fatty acid oxidation and assembly and secretion of VLDL are related to PTEN regulation. To understand the role of PTEN in regulating fatty acid oxidation and assembly and secretion of VLDL in dairy cow liver, we transfected a PTEN overexpression vector into dairy cow primary hepatocytes. Using these hepatocytes, we evaluated the effects and mechanisms of PTEN on the etiology of fatty liver disease of dairy cow. At least in part, PTEN could be a potential target for the gene treatment of cattle with fatty liver disease.

\section{Materials and Methods}

\section{Adenovirus overexpression vector construction}

Dairy cow PTEN full-length cDNA was generated by RT-PCR from the total RNA of cow liver tissue. The primers are listed in Table 1 . The purified PCR product was then directly subcloned into the multicloning site of the pMD-18T vector (TaKaRa, Otsu, Shiga, Japan). The gene was then digested by EcoR I and Hind III restriction enzymes and subcloned into the multicloning site of a pShuttle-GFP-CMV vector (Invitrogen, Carlsbad, US). The resulting plasmid, called pShuttle-GFP-PTEN, was then transferred to a pAdxsi vector (Invitrogen, Carlsbad, US), resulting in the pAdxsi-GFP-PTEN adenovirus plasmid. The pAdxsi-GFP-PTEN plasmid was transfected into 293 cells. After packaging, collecting and amplifying, the viral titer was $1.3 \times 10^{10} \mathrm{PFU} / \mathrm{ml}$. The Ad-GFP-pPTEN was stored at $-80^{\circ} \mathrm{C}$ for future experiments. 
Table 1. List of primers used in this paper.

\begin{tabular}{|c|c|c|}
\hline Genes & Primers used for PCR & Length of fragment (bp) \\
\hline \multirow{2}{*}{$\beta$-actin } & F:GTCATCACCATCGGCAATGAG & \multirow[t]{2}{*}{403} \\
\hline & R:GCTAACAGTCCGCCTAGAAGCA & \\
\hline \multirow{2}{*}{ ApoB } & F:GAT ACT CAG AAC GGA GCA AT & \multirow[t]{2}{*}{223} \\
\hline & R:GCA CCA ATC AGA TAA CAG GA & \\
\hline \multirow{2}{*}{ ApoE } & F:TCC TGA ATG ACC TGG GTG TTG & \multirow[t]{2}{*}{217} \\
\hline & R:TCT GTG GGT TGC CGT GGT G & \\
\hline \multirow{2}{*}{ MTP } & F:CAG TTT GCA GCC TTG GTT CTG & \multirow[t]{2}{*}{201} \\
\hline & R:TTC AAA AGC ACC GAG AGC GTT & \\
\hline \multirow{2}{*}{ LDLR } & F:GCT GTT CTG CCT TTC TCC TT & \multirow[t]{2}{*}{228} \\
\hline & R:ACT TTC TCC CCT GAC CCT TG & \\
\hline \multirow[t]{2}{*}{$\mathrm{HADH}$} & F: GGTCCACAGCACAGACTTGGT & \multirow[t]{2}{*}{151} \\
\hline & R: GGAATTGGCTAGGCTTGTGATC & \\
\hline \multirow{2}{*}{ PTEN } & F: AAGCTTATGAGAGACGGCGGCGG & \multirow[t]{2}{*}{139} \\
\hline & R: GGATCCTCAGACTTTTGTAATTTGTGTATGC & \\
\hline \multirow{2}{*}{ CPT } & F:GGTCAACAGCAACTACTACG & \multirow[t]{2}{*}{188} \\
\hline & R:TGAACATCCTCTCCATCTGG & \\
\hline \multirow{2}{*}{ ACSL } & F: TCGGAACTGAAGCCATCACC & \multirow[t]{2}{*}{173} \\
\hline & R: GCCTCGTTCCAGCAGATCAC & \\
\hline
\end{tabular}

Cell culture and transfection

Experiments were performed in accordance with the Guiding principles in the use of animals, adopted by the Chinese Association for Laboratory Animal Sciences. The study plan was approved by the Ethics Committee on the Use and Care of Animals, Jilin University (Changchun, China).

The caudate lobe of the liver was obtained through surgery liver excision from a one-day-old calf from a dairy farm located in Jilin Province, China (Laboratory dairy farm Jilin Agricultural University ). The calf was nursed care, and recovered finally. Calf hepatocytes were isolated by a modified two-step collagenase perfusion method established by our laboratory [20]. The cells were maintained in culture in a humidified atmosphere of $5 \% \mathrm{CO}_{2}$ at $37^{\circ} \mathrm{C}$. Hepatocytes obtained by modified two-step collagenase perfusion were intact and displayed good viability and active function. On the third day of primary culture, calf hepatocytes have optimal function. The cells were assigned to three groups: a control group (non-infected, $n=6$ ), a negative control group (infected with AD-GFP, $n=6$ ) and an AD-GFP-PTEN group (infected with AD-GFP-PTEN, $n=6$ ). Cells were covered by $2 \mathrm{ml}$ of serum-free medium. The adenoviruses were added to the medium at MOIs of 25,50,100, 150 and 200. The expression of GFP and the appearance of hepatocytes were observed by confocal scanning microscopy. The expression ratio of GFP was detected by flow cytometry (FCM).

\section{Quantitative real-time RT-PCR}

Total RNA was extracted using the using TRIzol reagent according to manufacturer's instructions (Invitrogen Corp, Carlsbad, CA) from cells 48 hours after transfection and quantified by an ultraviolet spectrophotometer. Three micrograms of total RNA were reverse-transcribed into cDNA using the BioRT one-step RT-PCR kit (BioFlux, Hangzhou, China). Two microliters of cDNA were used for relative quantitative PCR amplification using the standard Sybr GreenI PCR protocol provided by the manufacturer on an ABI PRISM 7000 quantitative real-time PCR instrument (Applied Biosystems, Foster City, CA). To evaluate the effect of PTEN in fatty acid synthesis, oxidation and assembly and the secretion of VLDL, PTEN, HADH, ACSL, LDLR, MTP, APOB, APOE and CPT I gene expression levels (primers are listed in Table 1) were measured in at least three independent experiments and normalized to the expression level of $\beta$-actin. Real-time PCR was conducted under the following conditions: initial denaturation at $94^{\circ} \mathrm{C}$ for $2 \mathrm{~min}, 35$ cycles of amplification (denaturation at $94^{\circ} \mathrm{C}$ for $10 \mathrm{~s}$, annealing at $60^{\circ} \mathrm{C}$ for $15 \mathrm{~s}$, and extension at $72^{\circ} \mathrm{C}$ for $30 \mathrm{~s}$ ), and extension at $72^{\circ} \mathrm{C}$ for $5 \mathrm{~min}$. 
Fig. 1. The expression of GFP in control group, AD-GFP-PTEN group after transfecting $48 \mathrm{~h}$ and the expression of GFP of several MOI. A: Expression of GFP in control group after transfecting 48h. B: Expression of GFP in AD-GFP-PTEN group after transfecting 48h. C:The expression of GFP of several MOI.

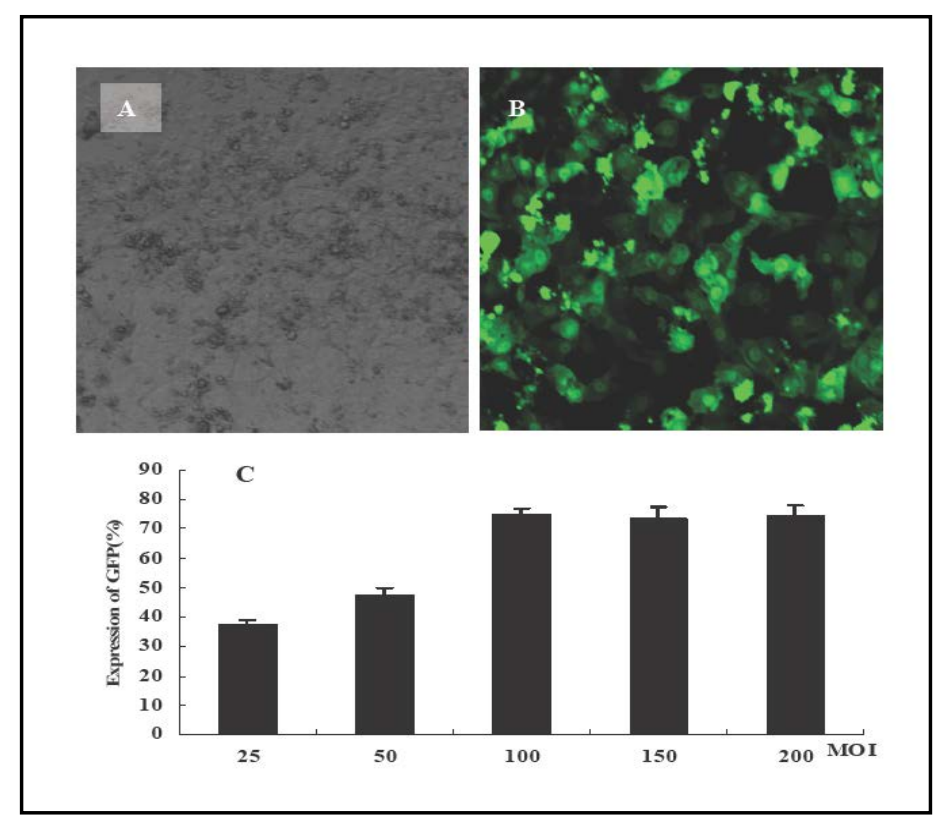

Western blot analysis

Forty-eight hours after transfection, cells were lysed in lysis buffer (50 mmol/l Tris-HCl pH 8.0; 0.150 $\mathrm{mmol} / \mathrm{l} \mathrm{NaCl} ; 1 \%$ Triton X-100; $100 \mu \mathrm{g} / \mathrm{ml} \mathrm{PMSF}$ ), and the protein concentration was measured using the BCA protein assay kit (Pierce, Rockford, US). PTEN, HADH, ACSL, LDLR, MTP, APOB, APOE and CPT I proteins were detected in hepatocytes. For western blot analysis, $30 \mu \mathrm{g}$ protein samples were boiled in $5 \times$ sample buffer for $5 \mathrm{~min}$ and then resolved by 10\% SDS-PAGE. Proteins were then transferred from gels to nitrocellulose membranes. After blocking, membranes were probed using first and then second antibodies (Santa Cruz Biotechnology, Santa Cruz, CA), and the resulting bands were detected by a DAB kit (Bioer, Hangzhou, China).

\section{Detection of TG and VLDL}

Forty-eight hours after transfection, cells and culture medium were collected. The quantity of TG in the cells was detected by the Triglyceride Detection Kit (Jiancheng, Nanjing, China). The VLDL in the culture medium was detected by the VLDL Detection Kit (Hailanji, Shanghai, China).

\section{Statistics}

All results are expressed as the means \pm standard error (SEM). Statistical analysis was carried out using the statistical analysis program SPSS 18.0 (SSPSinc.,Chicago,IL,USA). $p<0.05$ was considered to be significant.

\section{Results}

\section{AD-GFP transfection of hepatocytes and establishment of the best MOI}

Hepatocytes were transfected with AD-GFP at different MOIs (25, 50, 100, 150 and 200). After 48 hours, the positive rate of expression of AD-GFP was detected by FCM. When the MOI exceeded 100, the positive rate of expression of AD-GFP became stable. The expression of GFP in control and AD-GFP-PTEN groups was observed using confocal scanning microscopy (Fig. $1 \mathrm{~A}, \mathrm{~B}$ ). The positive rate of expression was approximately 75 percent by FCM (Fig. 1 C). MOI=100 was determined to be optimal according to apoptosis rates.

Overexpression of PTEN in hepatocytes

The PTEN gene was significantly overexpressed in hepatocytes transfected with ADGPF-PTEN $(p<0.01)$. However, PTEN expression in the control group and the negative control 


\section{Cellular Physiology $\quad$ Cell Physiol Biochem 2012;30:1005-1013 and Biochemistry

Fig. 2. The expression levels of mRNA and protein of PTEN, APOB100, CPTI, MTP, HADH, APOE, LDLR and ACSL was examined by qRT-PCR and western blot in heptocytes of newboned calf after transfecting ADGFP-PTEN 48h. A: The expression level of PTEN was examined by qRT-PCR in heptocytes of newboned calf after transfecting AD-GFP-PTEN 48h. B-H: The expression levels of APOB100, CPTI, MTP, HADH, APOE, LDLR and ACSL were examined by qRT-PCR in heptocytes of newboned calf after overexpressing PTEN 48h. I: The expression levels of APOB100, CPTI, MTP, HADH, APOE, LDLR, $\beta$-actin and ACSL were detected by Western blot in heptocytes of newboned calf after overexpressing PTEN 48h.
A

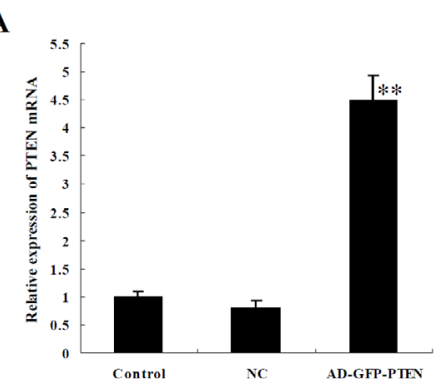

C

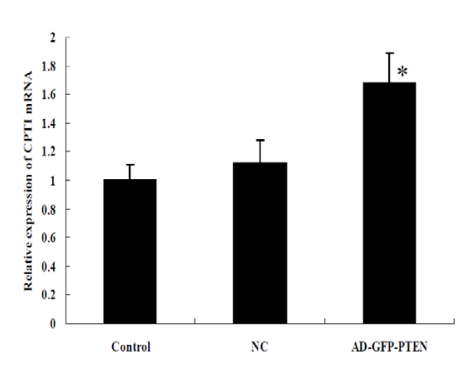

$\mathbf{E}$

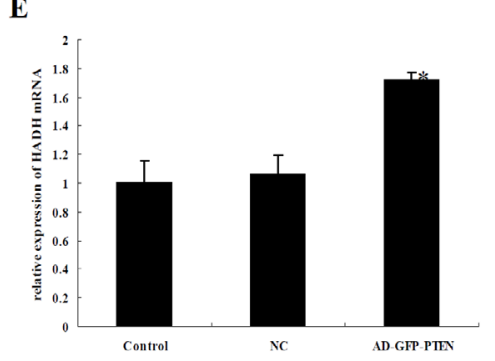

G

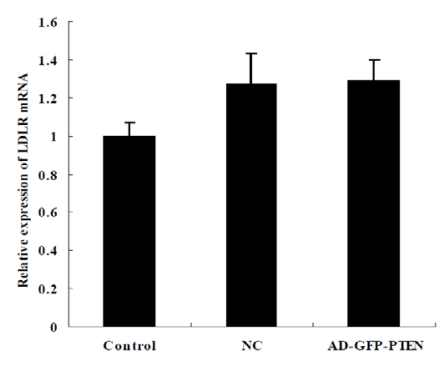

$\mathbf{F}$

H
D
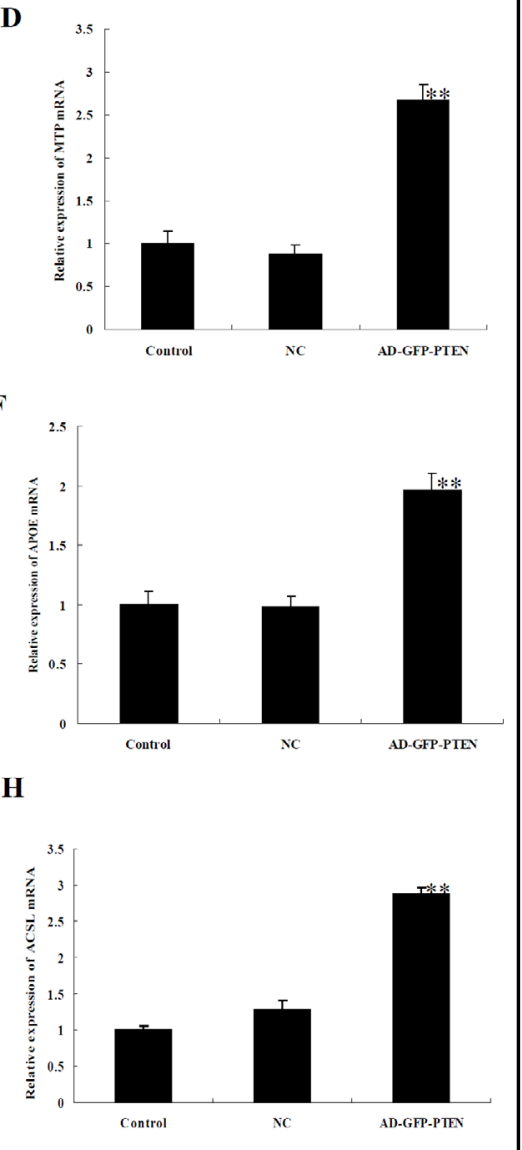

B

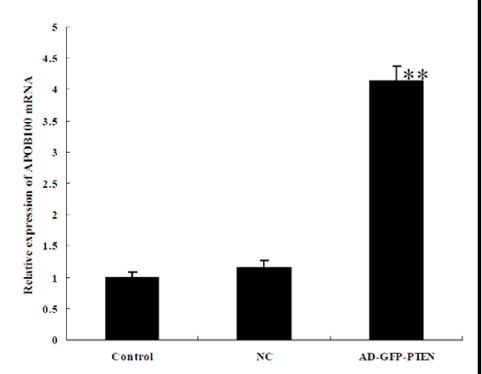

I

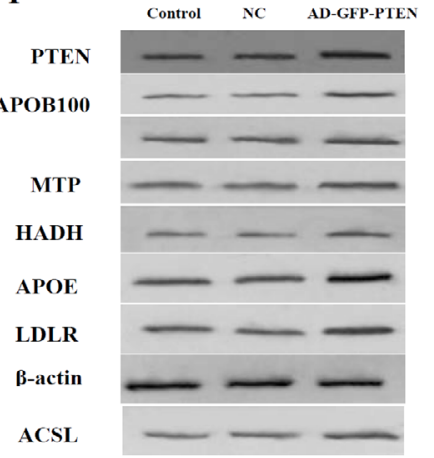

group was not significantly increased. These results were further confirmed by quantitative real-time RT-PCR (Fig. 2 A) and western blotting (Fig. 2 I). 
Fu/Deng/Yang et al.: Fatty Acid Oxidation and VLDL Assembly and Secretion Induced by the Overexpression of PTEN

Table 2. Concentration of TG in hepatocytes and VLDL in the culture medium after transfection $48 \mathrm{~h}$. (means \pm SEM). Superscript letters ${ }^{\mathrm{a}}$ and ${ }^{\mathrm{b}}$ indicate significant differences between the parameters of control group,negative control group and AD-GFP-PTEN group.

\begin{tabular}{lll}
\hline Groups & Concentration of TG $(\mathrm{mmoL} / \mathrm{L})$ & Concentration of VLDL $(\mathrm{mg} / \mathrm{mL})$ \\
\hline Control group & $0.5200 \pm 0.0500^{\mathrm{a}}$ & $6.1833 \pm 0.3800^{\mathrm{a}}$ \\
Negative control group & $0.4233 \pm 0.0577^{\mathrm{a}}$ & $9.2867 \pm 1.8277^{\mathrm{a}}$ \\
AD-GFP-PTEN group & $0.1367 \pm 0.0115^{\mathrm{b}}$ & $27.2467 \pm 6.0685^{\mathrm{b}}$ \\
\hline
\end{tabular}

Effect of fatty acid oxidation in hepatocytes by PTEN overexpression

CPT I, HADH and ACSL are the enzymes correlated with fatty acid oxidation. The expression of CPT I, HADH and ACSL mRNA and protein was increased in the AD-GFP-PTEN group compared to the control and negative control groups by overexpressing PTEN $(p<0.05)$ (Fig. 2 C, E, H, I). The expression of these enzymes in the control group and the negative control group was not significant $(p>0.05)$. These results indicated that the expression of key enzymes was up-regulated after overexpressing PTEN, consequently enhancing fatty acid oxidation.

Effect on assembly and secretion of VLDL in hepatocytes by PTEN overexpression

To investigate whether PTEN overexpression can change the assembly and secretion of VLDL in hepatocytes, the mRNA and protein levels of APOB, APOE, MTP and LDLR were detected. The expression of APOB $(p<0.01)$ and APOE $(p<0.05)$ in the AD-GFP-PTEN group was higher than in the control and negative control groups in several samples (Fig. 2 B, F, I), and the difference was statistically significant. Compared with the control and negative control groups, the expression of MTP in the PTEN overexpression group was increased $(p<0.01)$ (Fig. 2 D, I). The expression of LDLR mRNA and protein was slightly up-regulated (Fig. 2 G, I), but not significantly different ( $p>0.05$ ). The expression levels of three proteins (APOB, APOE, MTP)were not individually significantly different between the control group and the negative control group ( $p>0.05$ ). These results suggested that overexpressing PTEN could enhance the mRNA and protein expression of genes related to the assembly and secretion of VLDL.

\section{Changes of TG and VLDL}

To verify the effect of fatty acid metabolism regulated by overexpressing PTEN, the concentrations of TG and VLDL were detected in cells and culture medium, respectively. The concentration of VLDL in the AD-GFP-PTEN group was significantly higher in culture medium $(p<0.05)$, and TG concentration in the AD-GFP-PTEN group was lower in cells than the other groups $(p<0.01)$ (Table 2$)$.

\section{Discussion}

This in vitro study using PTEN-transfected primary hepatocytes showed that PTEN increased the expression of mRNA and protein from key genes related to fatty acid oxidation. We also show that overexpressing PTEN can enhance the expression of mRNA and protein from key genes related to the assembly and secretion of VLDL. We present several lines of evidence supporting the hypothesis that PTEN can enhance fat metabolism and decrease the incidence rate of fatty liver.

Recent studies have reported that PTEN could affect fat metabolism in PTEN knockout mice. In ten-week-old PTEN knockout mice, the liver enlarges and thins. At forty weeks, the liver enlarges further and whitens, with some lobes displaying serious fatty degeneration. 
TG content and cholesterol levels in the liver are also significantly increased in these mice. While Bechmann L reported that expression of PTEN was increased correlating with CD36 in humans non-alcoholic steatohepatitis [21]. The occur mechanism of fatty liver was regulated through defferent paths.

It has rarely been reported that PTEN affects the fat metabolism in the ruminant liver, in which lipoprotein lipase and hepatic lipase are deficient. Clearing TG is difficult through oxidation and hydrolysis; VLDL is the main TG clearance pathway [22]. Therefore, the assembly and transport of VLDL is the main factor influencing fatty liver of dairy cattle during the perinatal period. ApoB100, ApoE, MTP and soluble LDLR were proteins regulating fat transport in the liver $[23,24]$. APOB100 is the main constitutive protein and apolipoprotein of VLDL [11]. The activity and gene expression states of APOB100 and APOE can control the assembly and secretion rate of VLDL [25]. MTP participates in the pimelosis of APOB100 and the assembly of VLDL [26-28]. However, LDLR can inhibit secretion of APOB100. In this study, the expression levels of APOB100, APOE and MTP were up-regulated when PTEN was overexpressed. We also showed that the concentration of TG in cells decreased, the concentration of VLDL in culture medium increased in the AD-GFP-PTEN group. This result indicates that PTEN can enhance the assembly and secretion of VLDL by regulating the expression of these key proteins, thereby decreasing the deposition of TG. As reported by Taghibiglou $C$, stability of nascent apoB and enhanced expression of MTP increased the production of hepatic VLDL-apoB in fructose-fed hamsters [29]. Recent evidence also suggests a role for LDLR in the assembly and secretion of VLDL. LDLR prevents secretion of dense apoB100-containing lipoproteins [28]. In our results, the expression of LDLR mRNA was not significantly up-regulated, indicating that PTEN did not regulate the assembly and secretion of VLDL through the LDLR pathway.

CPT I plays a key role during the fatty acid oxidation process in mitochondria [30]. It is not only the rate-limiting enzyme in the first oxidation reaction [31], but CPT I can also regulate the entire fatty acid $\beta$-oxidation. CPT I catalyzes the transfer of acyl-CoA from a long-chain acyl-CoA ester to carnitine, forming acylcarnitine, which is then able to enter the mitochondria for $\beta$-oxidation [32]. ACSL is situated in the outer mitochondrial membrane. NEFA is sensitized to acyl-COA in the cytoplasm. Acyl-COA is transported into the mitochondria and becomes the substrate of $\beta$-oxidization or TG synthesis through FAS (fatty acid synthetase).

In this study, the expression levels of CPT I, HAD and ACSL were elevated in the ADGFP-PTEN group compared to the control and negative control groups during PTEN overexpression. After the expression of ACSL was elevated, the amount of sensitized NEFA increased. The amount of Acyl-CoA (the substrate of $\beta$-oxidization) entering into mitochondria was increased through the higher expression of CPT I, possibly enhancing $\beta$-oxidization. These results confirmed that PTEN could enhance $\beta$-oxidization through the regulation of key genes related to fat metabolism in the cow liver.

In summary, PTEN could enhance fatty acid oxidation and the assembly and secretion of VLDL. These results suggest that PTEN shows significant effects on the expression of key enzymes of dairy cow fatty acid metabolism, thereby reducing the deposition of fatty acid in the liver. PTEN gene therapy may be a new therapeutic strategy for fatty liver diseases of dairy cattle.

\section{Abbreviations}

PTEN (Phosphatase and tensin homolog); VLDL (very low density lipoprotein); CPT I (carnitine palmityl transferase I); ACSL (acyl-COA synthetase); HADH (3-hydroxy acylCoA dehydrogenase); ApoB100 (apolipoprotein B100); ApoE (apolipoprotein E); MTP (microsomal triglyceride transfer protein); LDLR (low-density lipoprotein receptor); NEB (hepatic lipidosis is negative energy balance); NEFA (nonesterified fatty acids); OAA (oxaloacetic acid); TG (Triglyceride). 
Fu/Deng/Yang et al.: Fatty Acid Oxidation and VLDL Assembly and Secretion Induced by the Overexpression of PTEN

\section{Acknowledgements}

This work was supported by a grant from the National Natural Science Foundation of China (No.30972224).

\section{References}

1 Kalaitzakis E, Panousis N, Roubies N, Giadinis N, Kaldrymidou E, Georgiadis M, Karatzias H: Clinicopathological evaluation of downer dairy cows with fatty liver. Can Vet J 2010;51:615-622.

2 Kalaitzakis E, Roubies N, Panousis N, Pourliotis K, Kaldrymidou E, Karatzias H: Clinicopathologic evaluation of hepatic lipidosis in periparturient dairy cattle. J Vet Intern Med 2007;21:835-845.

-3 Oikawa S, Mizunuma Y, Iwasaki Y, Tharwat M: Changes of very low-density lipoprotein concentration in hepatic blood from cows with fasting-induced hepatic lipidosis. Can J Vet Res 2010;74:317-320.

4 Pullen DL, Liesman JS, Emery RS: A species comparison of lover slice synthesis and secretion of triacylglycerol from nonesterified fatty acids in media. J Anim Sci 1990;68:1395-1399.

5 Herdt TH, Wensing T, Haagsman HP, van Golde LM, Breukink HJ: Hepatic triacylglycerol synthesis during a period of fatty liver development in sheep.J Anim Sci 1988;66:1997-2013.

6 Gerloff BJ, Herdt TH, Emery RS: Relationship of hepatic lipidosis to health and performance in dairy cattle. J Am Vet Med Assoc 1986;88:845-850.

7 Reid IM: Incidence and severity of fatty liver in dairy cows. Vet Rec 1980;107:281-284.

- Herdt TH: Fatty liver in dairy cows. Vet Clin North Am Food Anim Pract 1988;4:269-287.

9 Sun Y, Wang X, Li H, Wang Z, Zhang J: Effects of Dif ferent Energy Intake in the Dry Period on Abundance of Apol ipoprotein B100 mRNA in Liver of Periparturient Dairy Cows. Chin J Vet Sci 2006;26:320-322.

10 Sun Y, Wang Xg, Li H, Wang Z, Zhang J: Effects of Different Energy ingested Levels on Abundance of Apolipoprotein E mRNA in Liver of Periparturient Dairy Cows. Chin J Anim Vet Sci 2006;7:342-347.

11 Bernabucci U, Ronchi B, Basirico L, Pirazzi D, Rueca F, Lacetera N, Nardone A: Abundance of mRNA of Apolipoprotein B100, Apolipoprotein E,and Microsomal Triglyceride Transfer Protein in Liver from Periparturient Dairy Cows. J Dairy Sci 2004;87:2881-2888.

12 Kong G, Zhang J, Zhang S, Shan C, Ye L, Zhang X: Upregulated microRNA-29a by hepatitis B virus X protein enhances hepatoma cell migration by targeting PTEN in cell culture model. PLoS One 2011;6:e19518.

13 Li J, Yen C, Liaw D, Podsypanina K, Bose S, Wang SI, Puc J, Miliaresis C, Rodgers L, McCombie R, Bigner SH, Giovanella BC, Ittmann M, Tycko B, Hibshoosh H, Wigler MH, Parsons R: PTEN, a putative protein tyrosine phosphatase gene mutated in human brain, breast, and prostate cancer. Science 1997;275:1943-1947.

-14 Dasari VR, Kaur K, Velpula KK, Gujrati M, Fassett D, Klopfenstein JD, Dinh DH, Rao JS: Upregulation of PTEN in glioma cells by cord blood mesenchymal stem cells inhibits migration via downregulation of the PI3K/ Akt pathway. PLoS One 2010;5:e10350.

15 Selvendiran K, Kuppusamy ML, Bratasz A, Tong L, Rivera BK, Rink C, Sen CK, Kálai T, Hideg K, Kuppusamy P: Inhibition of vascular smooth-muscle cell proliferation and arterial restenosis by HO-3867, a novel synthetic curcuminoid, through up-regulation of PTEN expression. J Pharmacol Exp Ther 2009;329:959966.

16 Michael IP, Pampalakis G, Mikolajczyk SD, Malm J, Sotiropoulou G, Diamandis EP: Human tissue kallikrein 5 is a member of a proteolytic cascade pathway involved in seminal clot liquefaction and potentially in prostate cancer progression. J Biol Chem 2006;281:12743-12750.

17 Horie Y, Suzuki A, Kataoka E, Sasaki T, Hamada K, Sasaki J, Mizuno K, Hasegawa G, Kishimoto H, Iizuka M, Naito M, Enomoto K, Watanabe S, Mak TW, Nakano T: Hepatocyte-specific Pten deficiency results in steatohepatitis and hepatocellular carcinomas. J Clin Invest 2004;113:1774-1783.

18 Stiles B, Wang Y, Stahl A, Bassilian S, Lee WP, Kim YJ, Sherwin R, Devaskar S, Lesche R, Magnuson MA, Wu H: Liver-specific deletion of negative regulator Pten results in fatty liver and insulin hypersensitivity. Proc Natl Acad Sci USA 2004;101:2082-2087. 


\section{Cellular Physiology $\quad$ Cell Physiol Biochem 2012;30:1005-1013 and Biochemistry

19 He L, Hou X, Kanel G, Zeng N, Galicia V, Wang Y, Yang J, Wu H, Birnbaum MJ, Stiles BL: The Critical Role of AKT2 in Hepatic Steatosis Induced by PTEN Loss. Stiles Am J Pathol 2010;176:2302-2308.

20 Li X, Li X, Bai G, Chen H, Deng Q, Liu Z, Zhang L, Liu G, Wang Z: Effects of non-esterified fatty acids on the gluconeogenesis in bovine hepatocytes. Mol Cell Biochem 2012;359:385-388.

-21 Bechmann LP, Gieseler RK, Sowa JP, Kahraman A, Erhard J, Wedemeyer I, Emons B, Jochum C, Feldkamp T, Gerken G, Canbay A: Apoptosis is associated with CD36/fatty acid translocase upregulation in nonalcoholic steatohepatitis. Liver Int 2010;30:850-859.

22 Mason TM: The role of factors that regulate the synthesis and secretion of very-low-density lipoprotein by hepatocytes. Crit Rev Clin Lab Sci 1998;35:461-487.

23 Greenow K, Pearce NJ, Ramji DP: The key role of apolipoprotein E in atherosclerosis. J Mol Med (Berl) 2005;83:329-342.

24 Wang FB, Zhu CL, Liu X, Gao GS: HBV inhibits apoB production via the suppression of MTP expression. Lipids Health Dis 2011;10:207.

25 Ooi EM, Janus ED, Grant SJ, Sinclair LM, R Barrett PH: Effect of apolipoprotein E genotype on apolipoprotein B-100 metabolism in normolipidemic and hyperlipidemic subjects. J Lipid Res 2010;51:2413-2421.

26 Hahn SE, Goldberg DM: Factors affecting the regulation of apo B secretion by liver cells. J Clin Lab Anal 1995;9:431-449.

27 Liu Y, Manchekar M, Sun Z, Richardson PE, Dashti N: Apolipoprotein B-containing lipoprotein assembly in microsomal triglyceride transfer protein-deficient McA-RH7777 cells. J Lipid Res 2010;51:2253-2264.

28 Larsson SL, Skogsberg J, Björkegren J: The low density lipoprotein receptor prevents secretion of dense apoB100-containing lipoproteins from the liver. J Biol Chem 2004;279:831-836.

-29 Taghibiglou C, Carpentier A, Van Iderstine SC, Chen B, Rudy D, Aiton A, Lewis GF, Adeli K: Mechanisms of hepatic very low density lipoprotein overproduction in insulin resistance. Evidence for enhanced lipoprotein assembly, reduced intracellular ApoB degradation, and increased microsomal triglyceride transfer protein in a fructose-fed hamster model. J Biol Chem 2000;275:8416-8425.

30 Al-Aqeel AI, Rashed MS, Ruiter JP, Al-Husseini HF, Al-Amoudi MS, Wanders RJ: Carnitine palmityl transferase I deficiency. Saudi Med J 2001;22:1025-1029.

-31 Lee K, Kerner J, Hoppel CL: Mitochondrial carnitine palmitoyltransferase 1a (CPT1a) is part of an outer membrane fatty acid transfer complex. J Biol Chem 2011;286:25655-25662.

32 Linher-Melville K, Zantinge S, Sanli T, Gerstein H, Tsakiridis T, Singh G: Establishing a relationship between prolactin and altered fatty acid $\beta$-Oxidation via carnitine palmitoyl transferase 1 in breast cancer cells. BMC Cancer 2011;DOI: $\underline{10.1186 / 1471-2407-11-56}$. 